\title{
Chronic Psychotic Disorder Due to Steroid-Responsive Encephalopathy Associated with Hashimoto's Thyroiditis
}

To the Editor:

August 17, 2007

Hashimoto's thyroiditis is a common cause of thyroid disorder worldwide. This thyroiditis often progresses to hypothyroidism. Psychiatric disorders, especially depression, are commonly described in these patients even after correction of hypothyroidism. Herein we report on a case of a steroid responsive encephalopathy associated with Hashimoto's thyroiditis (SREHT) misdiagnosed as a primary chronic psychotic disorder.

\section{CASE REPORT}

A 37-year-old woman was admitted to our department with auditory hallucinations (denigrating voices) and persecutory ideas. Her physical examination revealed an altered mental status marked by impaired attention, disorientation and mental slowness. No motor neurological sign was noticed.

She was asymptomatic until 35 years of age when she developed a subacute paranoia with agitation. At that time she was admitted to a regional psychiatric hospital and a diagnosis of schizophrenia was made.

Over the next two years, she was treated with neuroleptics, including haloperidol and risperidone. Despite treatment she displayed psychotic outbursts requiring several hospital admissions. We hypothesized an organic mental disorder and a careful laboratory workup was performed. Serum levels of free thyroxine were mildly decreased $(0.7 \mathrm{ng} / \mathrm{dL}$; normal range: $0.8-1.8 \mathrm{ng} /$ $\mathrm{dL}$ ) and the serum levels of thyroid stimulating hormone were significantly increased $(13.1 \mathrm{mIU} /$ $\mathrm{mL}$; normal range: $0.3-5.0 \mathrm{~m}(\mathrm{U} / \mathrm{mL}$ ). High lev- els of thyroperoxidase (TPO) antibody $(1,742 \mathrm{IU} /$ $\mathrm{mL}$; normal range $<20 \mathrm{mlU} / \mathrm{mL}$ ) were also found. Other laboratory exams, including hemogram, ionogram, liver and renal function, serological studies for HIV and syphilis, were unremarkable. Neuroimaging studies were unrevealing as well as cerebrospinal fluid analysis.

Electroencephalogram demonstrated generalized slowing with episodes of triphasic waves, findings compatible with metabolic encephalopathy. The patient was treated with levothyroxine $0.1 \mathrm{mg} /$ day and prednisolone $100 \mathrm{mg} /$ day, that was gradually tapered. She evolved with complete remission of psychotic symptoms in parallel of the decline of TPO antibody titers in five months. In the follow-up, she had no need of antipsychotic treatment.

The final diagnosis of the patient was SREHT. SREHT is a rare encephalopathy encompassing neurological symptoms, such as seizures, myoclonus, tremor and less frequently focal deficits, as well as behavioral changes in association with increased serum levels of thyroid antibody. ${ }^{1,2}$ Patients may be in euthyroid state or with mild hypothyroidism. By definition the neuropsychiatric symptoms must improve with corticosteroid treatment. ${ }^{1,2}$

\section{CONCLUSION}

Psychotic symptoms, except visual hallucinations, are not common in SREHT. In a recent case series, Castillo and colleauges ${ }^{3}$ observed psychosis or paranoia in 5 out of 20 patients (25\%) seen during an 8-year period at the Mayo Clinic. Misdiagnosis is frequent, especially considering that routine laboratory investigations 
(not including TPO antibody) are often normal. Furthermore, symptoms tend to fluctuate over time and their severity is not related with TPO levels. ${ }^{3}$ The prominent psychotic features at presentation contributed to the misdiagnosis of the present case. Nevertheless, the relatively acute onset and the onset occurring after 30 years of age should had prompted careful investigation even in the absence of an altered mental status.

This case highlights the relevance of a high level of suspicion and a careful investigation of secondary causes of psychotic disorders.

Sincerely,

Felipe Filardi da Rocha, MD

Humberto Correa, MD

Antonio Lucio Teixeira, MD

\section{REFERENCES}

1. Chong J, Rowland L, Utiger R. Hashimoto encephalopathy: syndrome or myth? Arch Neurol. 2003:60:164-171.

2. Ferracci $C$, Carnevale A. The neurological disorder associated with thyroid autoimmunity. $J$ Neurol. 2006;253:975-98s.

3. Castillo P, Wooddruff B, Caselli R, et al. Steroid-responsive encephalopathy associated with autoimmune thyroiditis. Arch Neurol. 2006:63:197-202.

Dr. da Rocha is resident and Dr. Correa is associate professor of psychiatry, both in the Department of Psychiatry; and Dr. Teixeira is associate professor of neurology in the Department of Internal Medicine all at the School of Medicine at the Federal University of Minas Gerais in Belo Horizonte, Brazil.

Disclosure: The authors do not have an affiliation with or financial interest in any organization that might pose a conflict of interest.

Please send letters to the editor to: CNS Spectrums, c/o Eric Hollander, MD, 333 Hudson St., 7th Floor, New York, NY 10013; E-mail: vi@mblcommunications.com.

\section{Now Available Online at www.cnsspectrums.com CME-ACCREDITED SUPPLEMENT CME]} An expert review of clinical challenges in psychiatry

\section{The Complexity of ADHD: Diagnosis and Treatment of the Adult Patient with Comorbidities}

by Jeffrey H. Newcorn, MD, Margaret Weiss, MD, PhD, and Mark A. Stein, PhD, ABPP

To request a published supplement, please E-mail ks@mblcommunications.com

Supported by an educational grant from Eli Lilly and Company. 